\title{
Five cases of intracranial tuberculoma followed by serial computerised tomography
}

\author{
R. C. PEATFIELD A N D H. H. SH A W DON
}

From the Central Middlesex Hospital, London

SUMMARY Five cases of intracranial tuberculoma in Asian adults are described, all of whom presented with epilepsy. The computerised tomographic (CAT) scanner displayed the lesions particularly well, and their characteristics are described. Only two cases were explored surgically, but triple antituberculous chemotherapy has caused the scan abnormalities in all of them to resolve completely. It is concluded that Asian patients with lesions likely to be tuberculous should be given a trial of antituberculous drugs, and only explored if they should deteriorate clinically, or if the lesion should fail to respond on serial scans.

In 3533 brain necropsies in Leeds between 1910 and 1931, 264 space-occupying lesions were found, of which $34 \%$ were tuberculomas (Garland and Armitage, 1933). In Cushing's experience (1932), and in later clinical series from Europe and the United States of America, however, the proportion fell to less than 4\% (Obrador, 1959; Berlin, 1963; Maurice-Williams, 1972; Anderson and MacMillan, 1975). In developing countries, in contrast, $30 \%$ remains a typical figure (Asenjo et al., 1951; Ramamurthi and Varadarajan, 1961; Dastur and Desai, 1965; Dastur et al., 1968; Poltera and Templeton, 1973). Increasing immigration and improved diagnostic techniques both contribute to the frequency with which the condition is encountered and recognised (Anderson and MacMillan, 1975).

We report five cases of intracranial tuberculoma seen during one calendar year from a Regional Neurological Centre dealing with a large immigrant population. All the patients were adults from the Indian subcontinent. The CAT scan greatly simplified both initial diagnosis and follow-up of our patients, and we were able to show that regimes including rifampicin caused rapid and complete clinical and radiological response.

Address for reprint requests: Dr H. H. Shawdon, Department of Radiology, Central Middlesex Hospital, Acton Lane, London NW10 7NS.

Accepted 12 October 1978

\section{Case Reports (Table)}

CASE 1

A 45 year old Indian man who experienced three attacks of uncontrolled shaking of the right arm between September and December 1976 became dysphasic during one attack, but did not lose consciousness. The attacks were controlled by phenytoin. He had minimal weakness of his right arm and leg, but no other neurological abnormalities and the plantar responses were flexor. An isotope scan provided evidence of a focal lesion, and carotid arteriography demonstrated an avascular mass in the left frontoparietal region. At craniotomy in February 1977 (Mr R. Illingworth) a hard round mass was removed. The histology was typical of a tuberculoma, and acid-fast bacilli were grown from it. He was started on rifampicin, isoniazid, and ethambutol on the day after operation, and he made an uncomplicated recovery. A CAT scan in November 1977 (Fig. 1) showed only minimal left sided cerebral atrophy.

CASE 2

A 30 year old Sikh woman was admitted to another hospital in May 1977 after her second grand mal fit, the first having occurred two weeks before. There had been some twitching of the right hand in the second fit. She also gave a six month history of worsening left temporal headaches. There were no abnormal neurological signs, but an isotope scan suggested the presence of a low left parietal 


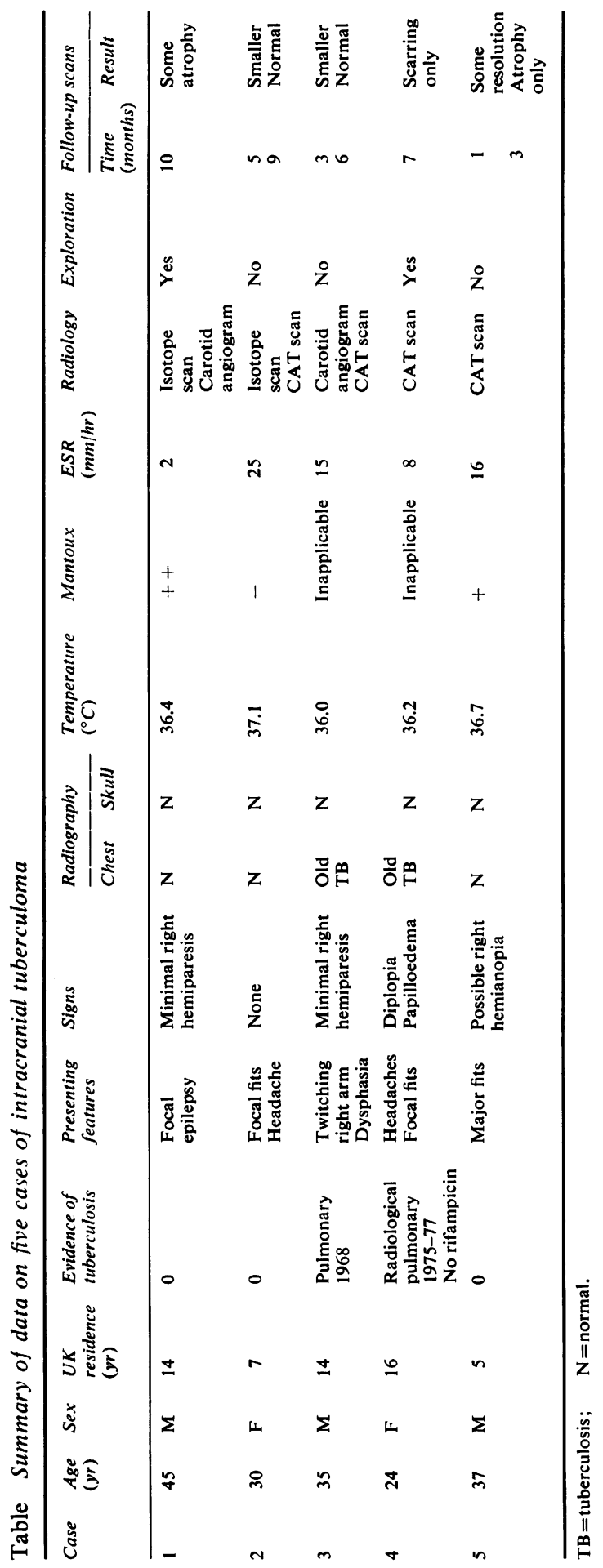




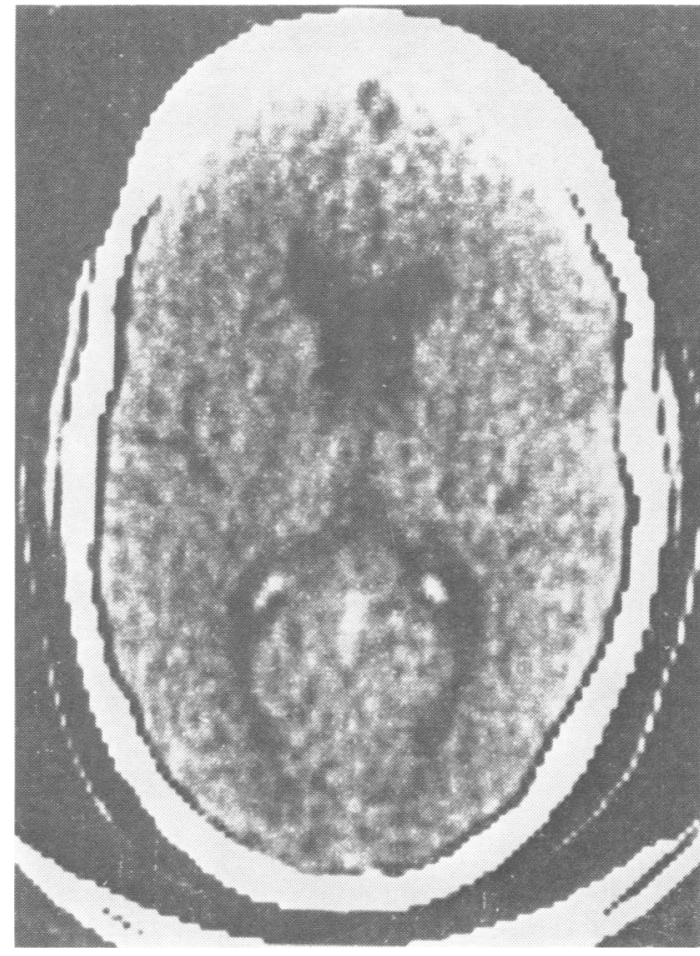

Fig. 1 Case 1. Unenhanced CAT scan in November 1977 showing minimal left sided atrophy. There was no enhancement with sodium iothalamate, but that scan showed some movement artefact.

lesion, and the CAT scanner showed an enhancing lesion posteriorly in the temporal lobe with considerable oedema (Fig. 2). A left carotid arteriogram confirmed a $40 \mathrm{~mm}$ avascular mass. She was started on rifampicin, isoniazid, and ethambutol in early June, but the development of neutropenia and hepatitis necessitated a change to ethambutol, isoniazid, streptomycin, and pyrizinamide, with phenobarbitone and phenytoin. She remained well: a second CAT scan in October 1977 showed a considerable reduction in the size of the lesion. and when repeated again in March 1978 it was normal (Fig. 3).

\section{CASE 3}

A 35 year old Indian man had received full chemotherapy with streptomycin, isoniazid, and pyrizinamide for pulmonary tuberculosis in 1968, and had been discharged from follow-up. He was admitted to another hospital in March 1977 with a fortnight's history of increasing frontal headache, and four episodes of uncontrolled twitching of the right arm, lasting for a few minutes, but followed by up to six hours of residual weakness and dysphasia. On

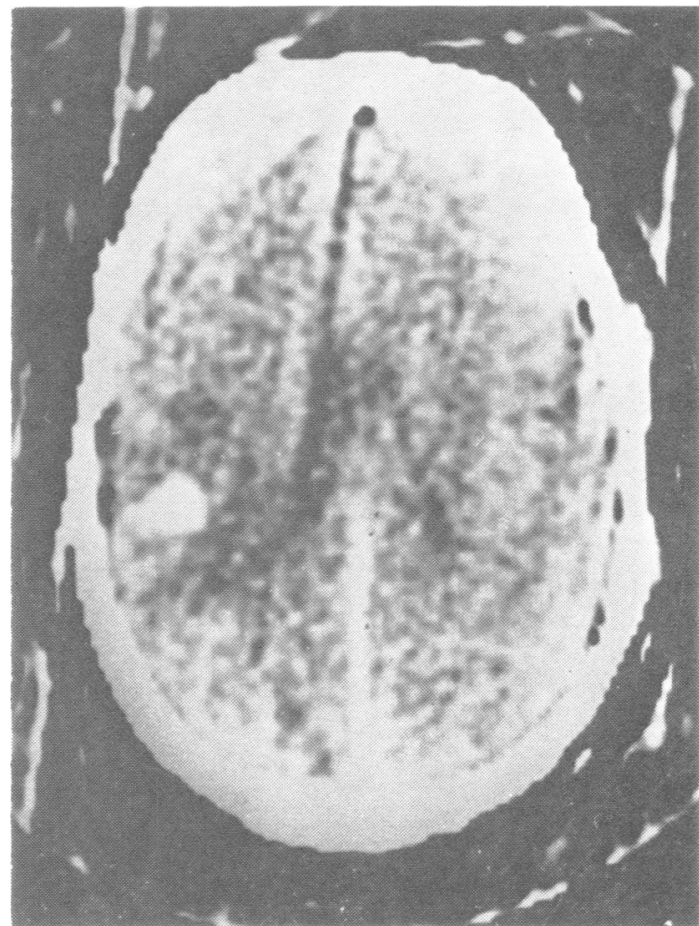

Fig. 2 Case 2. Enhanced scan in June 1977 showing a dense area of enhancement in the left temporal lobe surrounded by oedema.

examination there was a suggestion of a right hemiparesis but no other abnormal neurological signs. The EEG and isotope scan both suggested a left posterior frontal lesion. Left carotid arteriography showed an avascular mass with shift of midline structures by three millimetres, and a CAT scan (Fig. 4) showed a left parietal lesion with considerable enhancement with sodium iothalamate, and some oedema. He was started on rifampicin, isoniazid, and ethambutol in March 1977, and remained well at follow-up. A CAT scan in June 1977 showed a marked improvement, and it was within normal limits by October 1977 (Fig. 5). His EEG was normal in February 1978.

CASE 4

A 24 year old Indian woman who had pulmonary tuberculosis diagnosed radiologically was treated between May 1975 and May 1977 with streptomycin, ethambutol, and isoniazid. She had stopped drugs three months before admission. She gave a two year history of headaches, which had been worse during the previous four months, and she had had an ill-defined "blackout" in 1973. Two weeks before the birth of a child in May 1977, and 


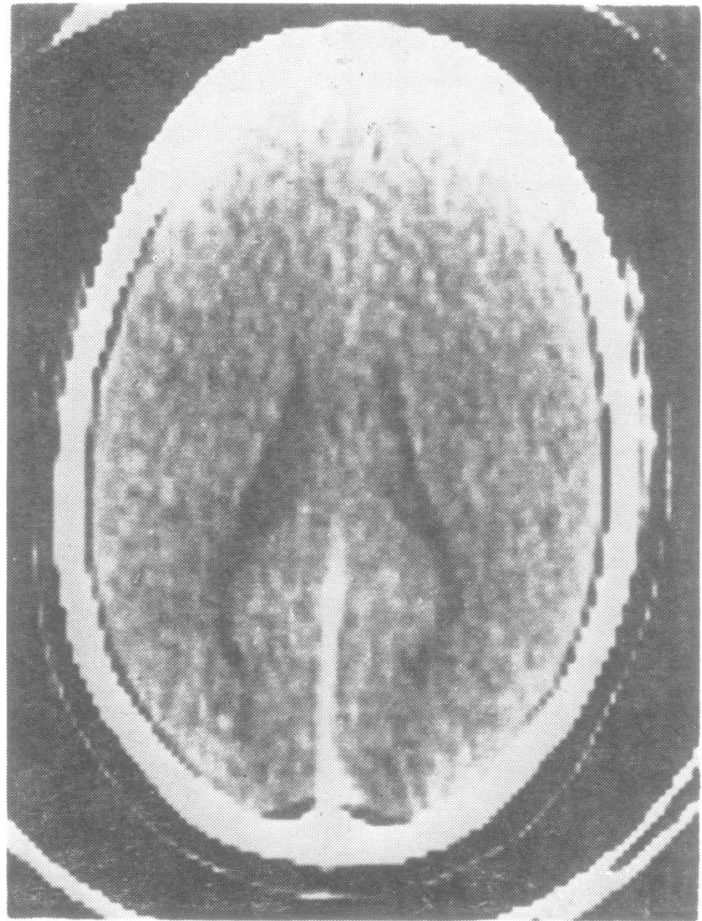

Fig. 3 Case 2. Repeat enhanced scan in March 1978 now shows no abnormality (same scan cut as Fig. 2).

again immediately after it, she experienced flashing lights in the left field of vision followed by loss of consciousness. She also gave a one week history of diplopia. On examination there was marked bilateral papilloedema and a bilateral sixth nerve paresis, but there were no other neurological signs. The CAT scan showed a right temporo-occipital lesion (Fig. 6) which was completely excised at craniotomy (Mr M. Rice Edwards). The histology was typical of tuberculosis, but acid-fast bacilli were not seen or cultured. Rifampicin, isoniazid, and ethambutol were started, and she made an uncomplicated recovery. The CAT scan was repeated in January 1978 and showed neurosurgical clips and some scarring, but no other abnormality and no shift of midline structures.

\section{CASE 5}

A 37 year old Indian man had been deaf since birth and had tinnitus in the right ear. He was seen at another hospital in September 1977, after a possible epileptic fit, but was not followed up. He was admitted to this hospital after a definite epileptic fit without focal features at work in December 1977. On examination there was no

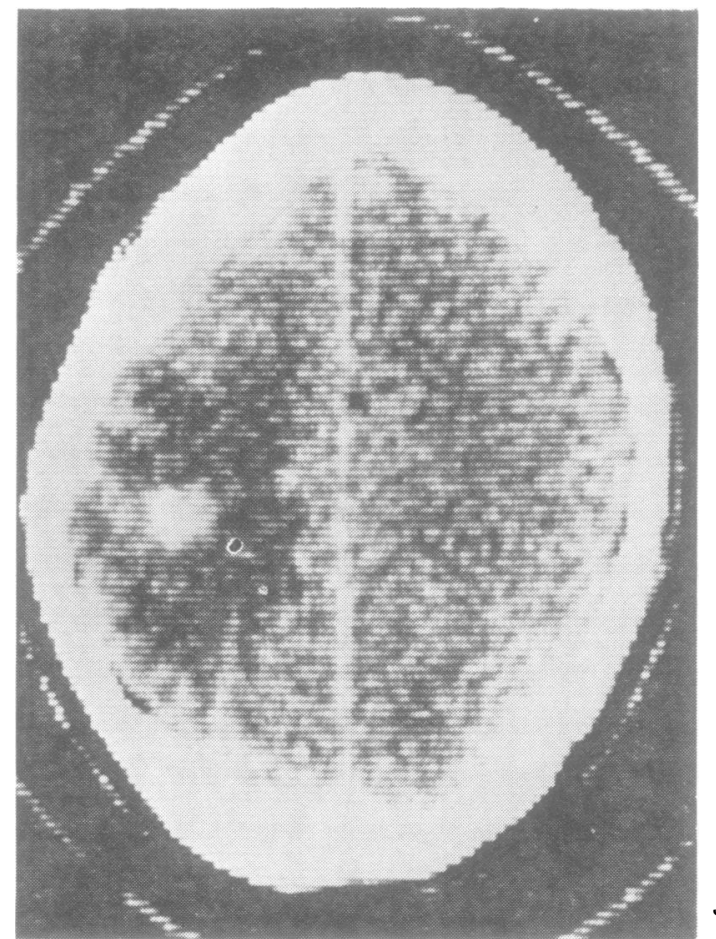

Fig. 4 Case 3. Enhanced scan in March 1977 showing a densely enhancing lesion in the left parietal lobe with considerable adjacent oedema.

significant neurological abnormality, though he had left sided chronic suppurative otitis media. His corrected visual acuity was normal, but formal visual field charting demonstrated a non-congruous homonymous field defect on the right. His EEG showed slow components over the left temporal region on hyperventilation only, but the CAT scan (Fig. 7) showed multiple areas of dense enhancement in the right occipital lobe, with oedema extending throughout the right hemisphere. He was started on rifampicin, isoniazid, and ethambutol, and a repeat scan in February 1978 showed some resolution. A further scan in April 1978 (Fig. 8) showed atrophic changes only, without any enhancement.

\section{Discussion}

All our cases were adult Indians, and all presented with epilepsy, which was focal in four of the five cases. Only two of the five patients complained of headache, and only one patient had papilloedema. Papilloedema was present in $80 \%$ of the 145 patients of Asenjo et al. (1951) and 93\% of the 107 patients of Dastur and Desai (1965). It seems 


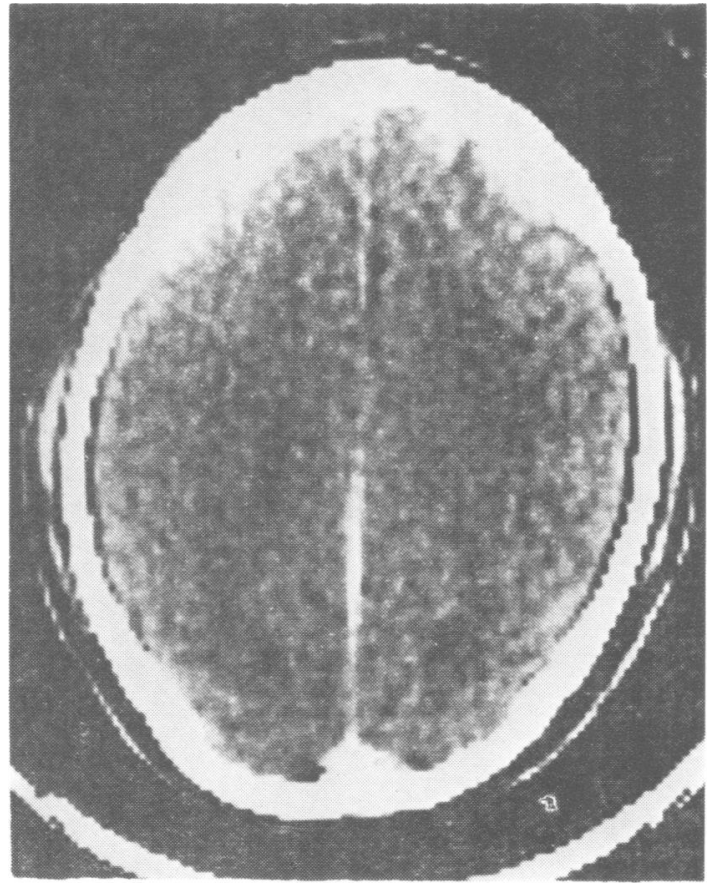

Fig. 5 Case 3. Enhanced scan in October 1977 shows no abnormality (same scan cut as Fig. 4).

probable that adults with epilepsy are investigated earlier in developed countries with greater neurodiagnostic facilities. Apart from this, the presenting features were similar to those in previous series (Sibley and O'Brien, 1956; Obrador, 1959; Dastur and Desai, 1965; Anderson and MacMillan, 1975). A history of treated tuberculous infection elsewhere in the body, a positive Mantoux test, and elevations of the temperature or ESR seem of limited value in distinguishing tuberculosis from other space-occupying lesions (Obrador, 1959; Dastur and Desai, 1965; Maurice-Williams, 1972; Anderson and MacMillan, 1975). The patients had been in Britain for an average of $\mathbf{1 1 . 2}$ years, which contrasts with the finding that pulmonary tuberculosis is most common in newly arrived Indian immigrants (British Thoracic and Tuberculosis Association, 1975).

Plain skull radiographs are unlikely to prove useful unless shift of a calcified pineal gland can be identified. Calcification of intracranial tuberculomas is rare, occurring in only $6 \%$ of the large series of Ramamurthi and Varadarajan (1961), and even less often in other series (Garland and Armitage, 1933; Asenjo et al., 1951; Berlin, 1963; Dastur, 1972). Isotope scanning, in contrast, seems a very useful technique (Anderson and MacMillan,

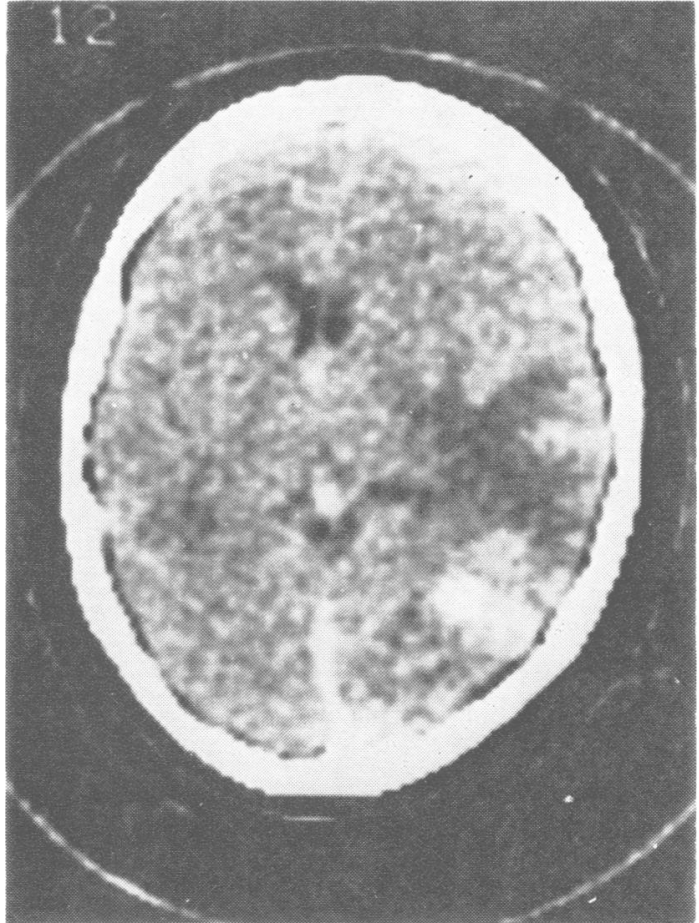

Fig. 6 Case 4. Enhanced CAT scan in July 1977 showing a large right parieto-occipital enhancing lesion with an even larger area of oedema extending forward from it. There is appreciable displacement of midline structures.

1975). The CAT scanner, particularly after enhancement with intravenous sodium iothalamate (Conray 420 ) seems even more sensitive, especially in the discovery of smaller or multiple lesions. There have been few published descriptions of the CAT scan appearances of cerebral tuberculoma (Claveria et al., 1976; Leilbrock et al., 1976; Szper, 1976). Two of our cases were seen initially before our CAT scan was commissioned, though case 3 was sent to another hospital for a scan after angiography. The oedema of ten surrounding the lesion is clearly shown, though previous authors have remarked that there is less angiographic displacement than the size of the tumour would suggest (Dastur and Desai, 1965). Serial scans are of particular value in following the response of the patient to chemotherapy after discharge from hospital, and a marked improvement can be expected within three months. The finding of an avascular mass at angiography, often with narrowed blood vessels in the vicinity, is now well described (Dastur and Desai, 1965; Dastur, 1972). The lack of angiographically demonstrable blood 


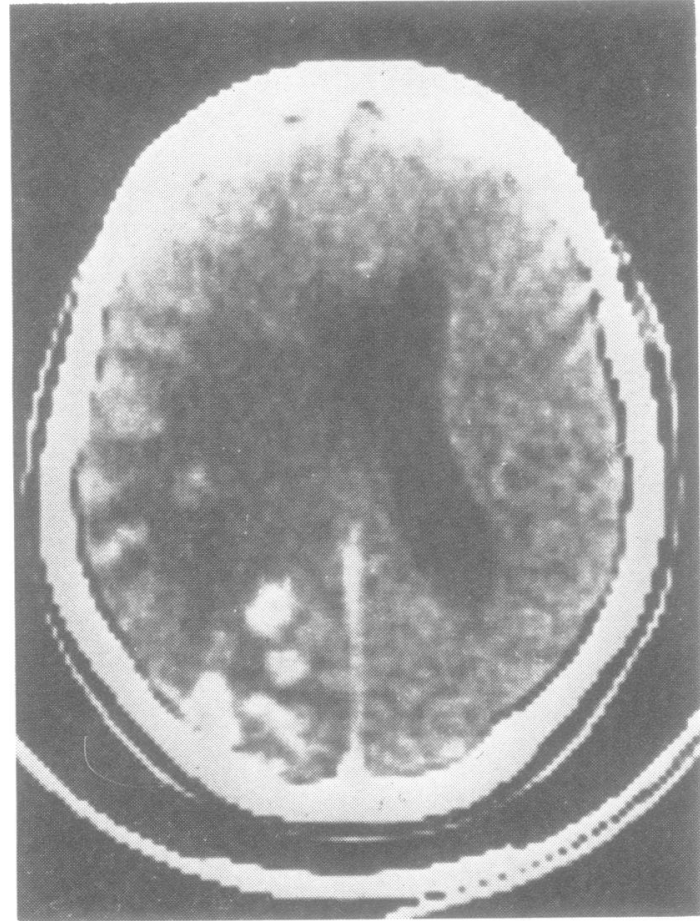

Fig. 7 Case 5. Enhanced scan in January 1978 showing multiple enhancing lesions in the left occipital and parietal lobes, oedema throughout the left hemisphere and significant midline shift.

vessels contrasts with the dense enhancement of the lesion in the CAT scan after intravenous iothalamate, which probably reflects increased vascular permeability (Kramer et al., 1975).

Early attempts at surgical extirpation were almost invariably followed by fatal tuberculous meningitis (Garland and Armitage, 1933; Asenjo et al., 1951; Sibley and O'Brien, 1956; Obrador, 1959), and Cushing (1932) recommended decompression without excision of the tuberculoma. With the advent of antituberculous chemotherapy this risk was greatly reduced, and many series of cases treated successfully by surgical exploration combined with pre- and postoperative chemotherapy have been reported (Sibley and O'Brien, 1956; Dastur and Desai, 1965; Maurice-Williams, 1972; Anderson and MacMillan, 1975). Indian authors have recommended that in developing countries most patients with uncomplicated intracranial mass lesions should receive a trial of antituberculous chemotherapy, with exploration only for patients who failed to respond symptomatically after two weeks (Ramamurthi and Natarajan, 1960; Ramamurthi and Varadarajan, 1961; Dastur,

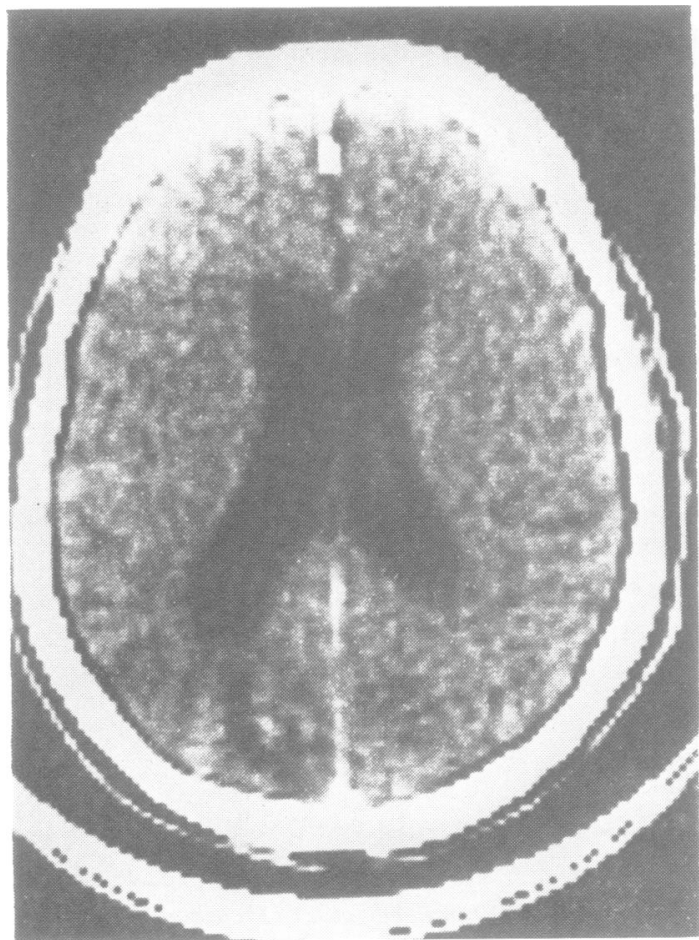

Fig. 8 Case 5. Enhanced scan in A pril 1978 showing some atrophy, predominantly of the left occipital lobe, but no areas of enhancement and no shift of midline (same scan cut as Fig. 7).

1972). Roedenbeck (1962) presents evidence that even patients with raised intracranial pressure can be treated with drugs alone. Two of our patients were explored surgically-case 4 because of evidence of raised intracranial pressure, and case 1 because tuberculoma was not considered the most likely diagnosis. Both these patients have done well on drugs started as soon as the histological diagnosis was available. The remaining three patients have been treated with rifampicin, isoniazid, and ethambutol without histology, and we take the complete clinical and radiological resolution in all of them as evidence that the lesions are tuberculous (Ramamurthi and Varadarajan. 1961).

Many reported cases have multiple tuberculomas $(54 \%$ in the necropsy series of Garland and Armitage (1933), $34 \%$ in the series of Asenjo et al. (1951), and one in this series), and chemotherapy is then the only available treatment. One case has been described in which a patient with miliary tuberculosis developed tuberculomas while on irregular isoniazid and ethambutol without rifampicin (Thrush and Barwick, 1974), but we 
anticipate that nine months of triple chemotherapy including rifampicin will be curative. even for relapses, as it seems to be in other organs (Fox. 1977). It is planned to treat case 2 for two years.

It is clear that tuberculoma is a significant cause of focal epilepsy in Asian adults, and is suggested by a lesion in the CAT scan which enhances densely with sodium iothalamate and may be surrounded by disproportionate oedema. In all such cases a trial of antituberculous chemotherapy may result in clinical improvement and cause resolution of the scan abnormality, thus eliminating the need for neurosurgical exploration.

We are particularly grateful to $\mathrm{Mr} \mathrm{R}$. D. Illingworth, Mr J. M. Rice Edwards, Dr J. C. Phemister, Dr L. S. Lange, and Dr M. W. McNicol for permission to report on their patients, and for assistance with the preparation of this paper. Figure 4 was supplied by courtesy of Dr J. Ambrose of Atkinson Morleys Hospital. The manuscript was typed by Miss E. Bell and Mrs P. Jennings.

\section{References}

Anderson, J. M., and MacMillan, J. J. (1975). Intracranial tuberculoma-an increasing problem in Britain. Journal of Neurology, Neurosurgery, and Psychiatry, 38, 194-201.

Asenjo, A., Valladares, H., and Fierro, J. (1951). Tuberculomas of the brain. Report of 159 cases. Archives of Neurology (Chicago), 65, 146-160.

Berlin, L. (1963). Tuberculoma of the brain. American Journal of Roentgenology, 90, 1185-1192.

British Thoracic and Tuberculosis Association (1975). Tuberculosis among immigrants related to length of residence in England and Wales. British Medical Journal, 3, 698-699.

Claveria, L. E., du Boulay, G. H., and Moseley, I. F. (1976). Intracranial infections: investigation by computerized axial tomography. Neuroradiology, 12, 59-71.

Cushing, H. (1932). Intracranial Tumours, pp. 114117. Charles C. Thomas: Springfield, Illinois.
Dastur, H. M. (1972). A tuberculoma review with some personal experiences. Ncurology India, 20, $111-126$.

Dastur, H. M., and Desai, A. D. (1965). A comparative study of brain tuberculomas and gliomas based upon 107 case records of each. Brain, 88, 375-396.

Dastur, D. K., Lalitha, V. A., and Prabhakar, V. (1968). Pathological analysis of intracranial spaceoccupying lesions in 1000 cases including children. Journal of the Neurological Sciences, 6, 575-592.

Fox, W. (1977). The modern management and therapy of pulmonary tuberculosis. Proceedings of the Royal Society of Medicine, 70, 4-15.

Garland, H. G., and Armitage, G. (1933). Intracranial tuberculoma. Journal of Pathology and Bacteriology, 37, 461-471.

Kramer, R. A., Janetos, G. P., and Perlstein, G. (1975). An approach to contrast enhancement in computed tomography of the brain. Radiology, 116, 641-647.

Leibrock, L., Epstein, M. H., and Rybock, J. D. (1976). Cerebral tuberculoma localized by EMI scan. Surgical Neurology, 5, 305-306.

Maurice-Williams, R. S. (1972). Tuberculomas of the brain in Britain. Postgraduate Medical Journal, 48, $678-681$.

Obrador, S. (1959). Intracranial tuberculomas: a review of 47 cases. Neurochirurgia, 1, 150-157.

Poltera, A. A., and Templeton, A. C. (1973). Intracranial tuberculosis in Uganda: a post-mortem survey. African Journal of Medical Sciences, 4, 34?349.

Ramamurthi, B., and Natarajan, M. (1960). Treatment of tuberculoma of the brain in children. Indian Journal of Child Health, 9, 193-197.

Ramamurthi, B., and Varadarajan, M. G. (1961). Diagnosis of tuberculomas of the brain. Journal of Neurosurgery, 18, 1-7.

Roedenbeck, S. D. (1962). Tuberculomas of the nervous system in children. World Neurology, 3, 55-64.

Sibley, W. A., and O'Brien, J. L. (1956). Intracranial tuberculomas-a review of clinical features and treatment. Neurology (Minneapolis), 6, 157-165.

Szper, I. (1976). Case report: tuberculoma. Illinois Medical Journal, 150, 593-596.

Thrush, D. C., and Barwick, D. D. (1974). Three patients with intracranial tuberculomas with unusual features. Journal of Neurology, Neurosurgery, and Psychiatry, 37, 566-569. 